\section{International Scientific Journal Theoretical \& Applied Science}

\author{
p-ISSN: 2308-4944 (print) e-ISSN: 2409-0085 (online) \\ Year: $2015 \quad$ Issue: $11 \quad$ Volume: 31 \\ Published: $30.11 .2015 \quad$ http://T-Science.org
}

SECTION 2. Applied mathematics. Mathematical modeling.
Sagat Zhunisbekov

doctor of technical Sciences, Professor, academician of the National Engineering Academy of the Republic of Kazakhstan, prorector of Taraz state University, Kazakhstan

Alexandr Shevtsov candidate of technical sciences, member of PILA (USA), Department of «Mathematics», Deputy Director on Science of faculty of information technologies, automation and telecommunications, Taraz state University, Kazakhstan Shev_AlexXXXX@mail.ru

\title{
ABOUT ONE INTERPOLATION MODEL PREDICTED VALUES
}

Abstract: The article provides one way analysis of projected data, based on the method of partitioning of the graph and interpolation of functions by splines.

Key words: spline, interpolation, projected data.

Language: English

Citation: Zhunisbekov S, Shevtsov AN (2015) ABOUT ONE INTERPOLATION MODEL PREDICTED VALUES. ISJ Theoretical \& Applied Science 11 (31): 185-191.

Soi: http://s-o-i.org/1.1/TAS-11-31-30 Doi: crossef http://dx.doi.org/10.15863/TAS.2015.11.31.30

This problem arose as a result of analysis of graphs and of discovering inaccurate data published in [1].

Analysis of the Observed data is known, but Estimated by the authors alleged their statement is obtained by interpolation of Observed data. But we discovered that it is not so. The calculation is true only for the first 4 points, and when you try to predict the future points of forecast Estimated wrong.

The process of analysis and design of interpolation functions is shown in Fig.1-4.

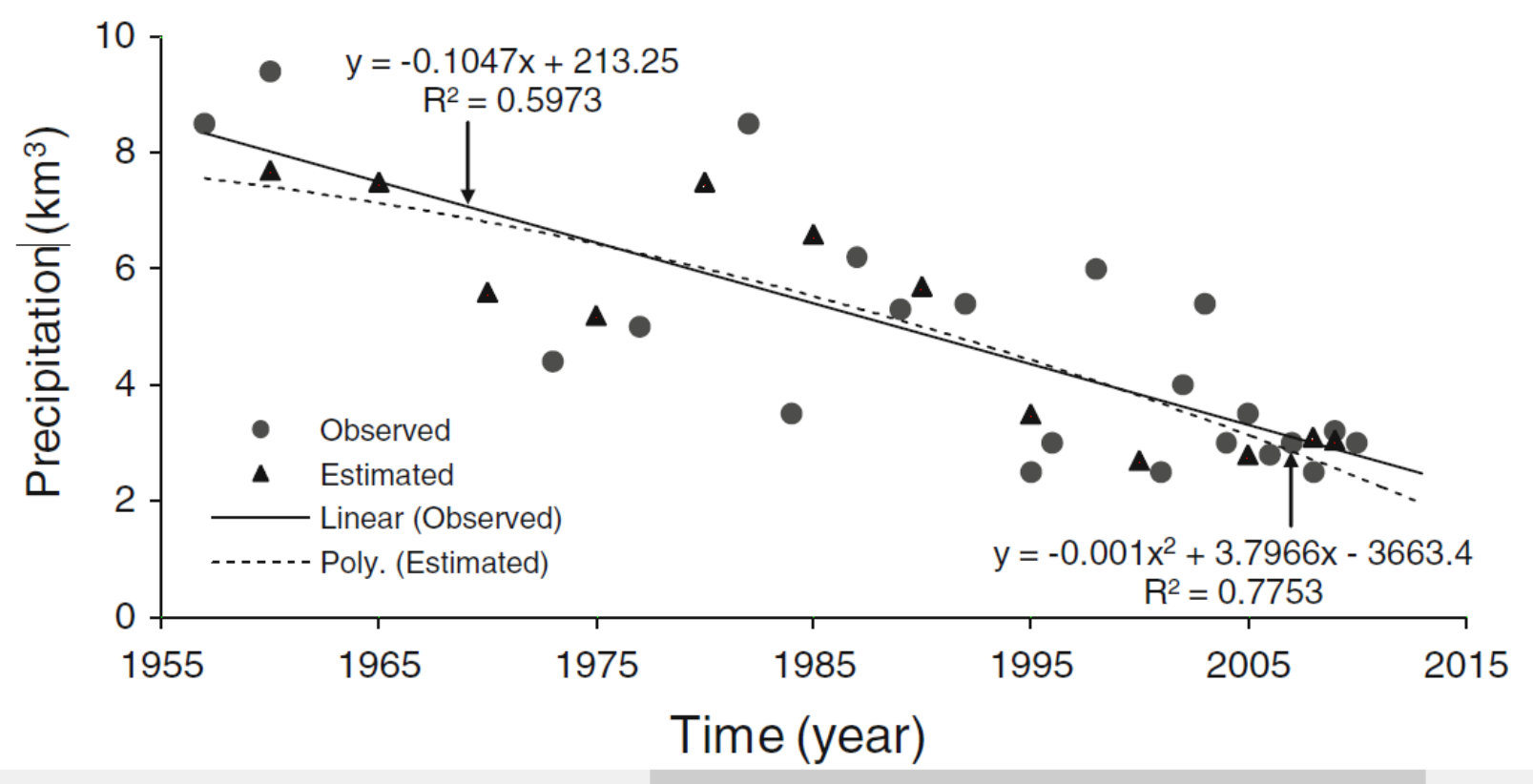

Figure 1 - Source data. 


\begin{tabular}{l|lrl|l|ll} 
& ISRA (India) & $=\mathbf{1 . 3 4 4}$ & SIS (USA) & $=\mathbf{0 . 9 1 2}$ & ICV (Poland) & $=\mathbf{6 . 6 3 0}$ \\
Impact Factor: & ISI (Dubai, UAE) $=\mathbf{0 . 8 2 9}$ & PUHL (Russia) $=\mathbf{0 . 1 7 9}$ & PIF (India) & $=\mathbf{1 . 9 4 0}$ \\
& GIF (Australia) & $\mathbf{0 . 5 6 4}$ & ESJI (KZ) & $=\mathbf{1 . 0 4 2}$ & & \\
& JIF & $\mathbf{1 . 5 0 0}$ & SJIF (Morocco) $=\mathbf{2 . 0 3 1}$ & & \\
\hline
\end{tabular}

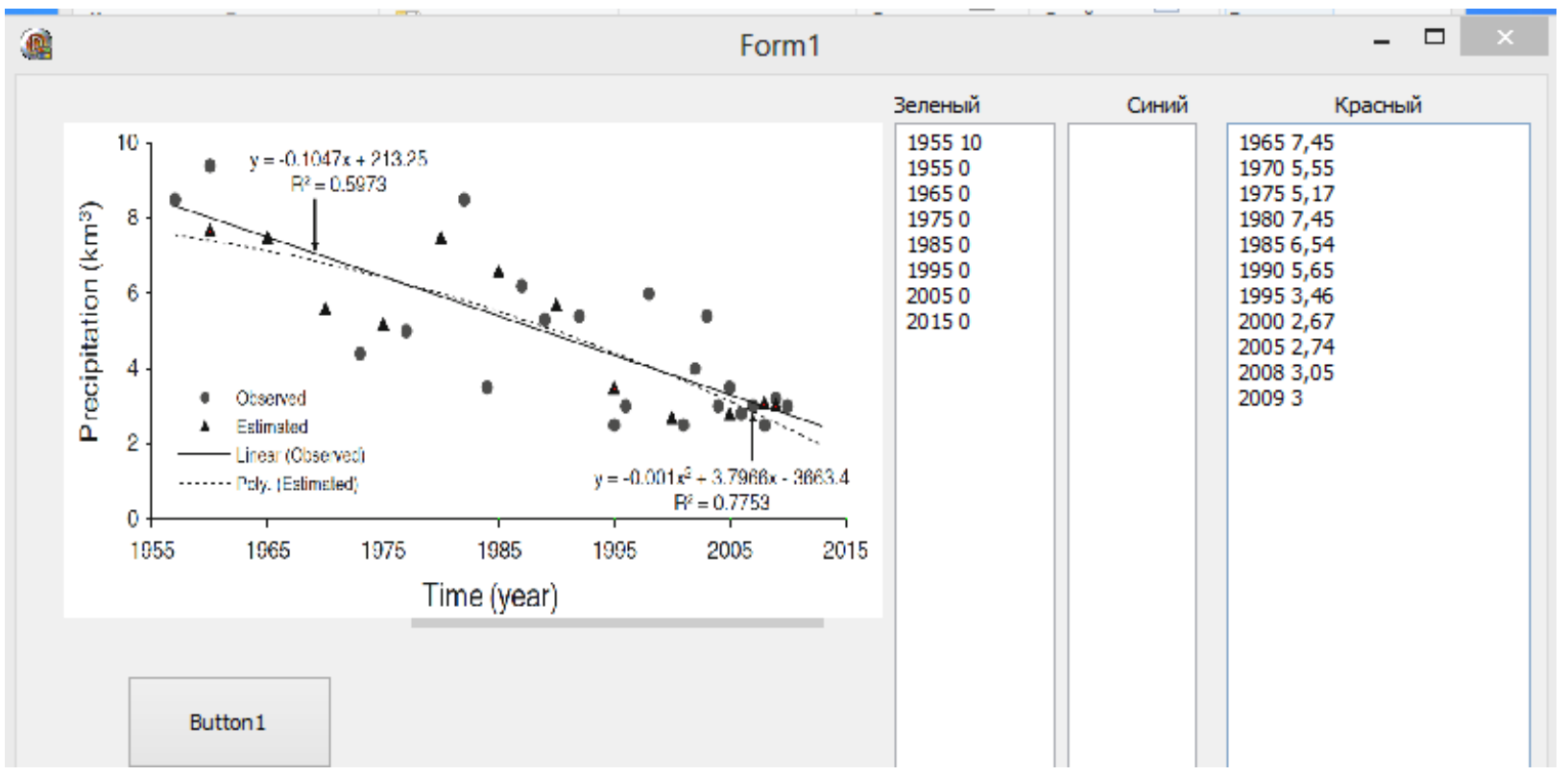

Figure 2 - Chart analysis and retrieval.

restart; readlib(spline) $: \mathrm{X}:=\mathrm{X}^{\prime}: \mathrm{Y}:=\mathrm{Y}^{\mathrm{Y}}$ ' :

$\mathrm{x}:=0,1,2,3,4,5,6,7,8,9,10,11$ :

$\mathrm{X} 1:=1960,1965,1970,1975,1980,1985,1990,1995,2000,2005,2008,2009$ :

$\mathrm{Y}:=15.87,26.14,10.35,3.01,1.67,1.09,7.77,6.68,3.59,4.76,5.01,4.76:$

$f:=[[X 1[n+1], Y[n+1]] \quad S n=0 \ldots 11]:$

fC:=spline $([\mathrm{X} 1],[\mathrm{Y}], \mathrm{x}, \mathrm{Cubic})$;

plot $([\mathrm{f}, \mathrm{fc}], \mathrm{x}=1955 \ldots 2010, \mathrm{y}=0.40$, style=[point, line, line, line] , color= $\mathrm{red}, \mathrm{gr}$ een,blue, red] , thickness=2, symbol=circle);

Figure 3 - Analysis of data on Maple and the construction of spline functions..

$f c=\left\{\begin{array}{cc}7.45-2.052594234 x+.1525942425 x^{3} & x<1 \\ 6.845565451-.239290574 x-1.81330366 x^{2}+.757028796 x^{3} & x<2 \\ 29.22747107-33.81214898 x+14.97312554 x^{2}-2.040709405 x^{3} & x<3 \\ -67.87852262+63.29384471 x-17.39553904 x^{2}+1.555808881 x^{3} & x<4 \\ 93.93493230-58.06624649 x+12.94448377 x^{2}-.972526352 x^{3} & x<5 \\ -154.4179515+90.94548392 x-16.85786232 x^{2}+1.014296721 x^{3} & x<6 \\ 147.7568292-60.14190647 x+8.323369414 x^{2}-.3846605983 x^{3} & x<7 \\ 21.18764135-5.897968794 x+.5742354584 x^{2}-.01565421941 x^{3} & x<8 \\ 101.6066454-36.05509532 x+4.343876274 x^{2}-.1727225868 x^{3} & x<9 \\ -101.9790772+31.80681221 x-3.196335674 x^{2}+.1065445225 x^{3} & \text { otherwise }\end{array}\right.$




\begin{tabular}{l|lrl|l|ll} 
& ISRA (India) & $=\mathbf{1 . 3 4 4}$ & SIS (USA) & $=\mathbf{0 . 9 1 2}$ & ICV (Poland) & $=\mathbf{6 . 6 3 0}$ \\
Impact Factor: & ISI (Dubai, UAE) $=\mathbf{0 . 8 2 9}$ & PUHU (Russia) $=\mathbf{0 . 1 7 9}$ & PIF (India) & $=\mathbf{1 . 9 4 0}$ \\
& GIF (Australia) & $\mathbf{0 . 5 6 4}$ & ESJI (KZ) & $=\mathbf{1 . 0 4 2}$ & & \\
& JIF & $=\mathbf{1 . 5 0 0}$ & SJIF (Morocco) $=\mathbf{2 . 0 3 1}$ & &
\end{tabular}

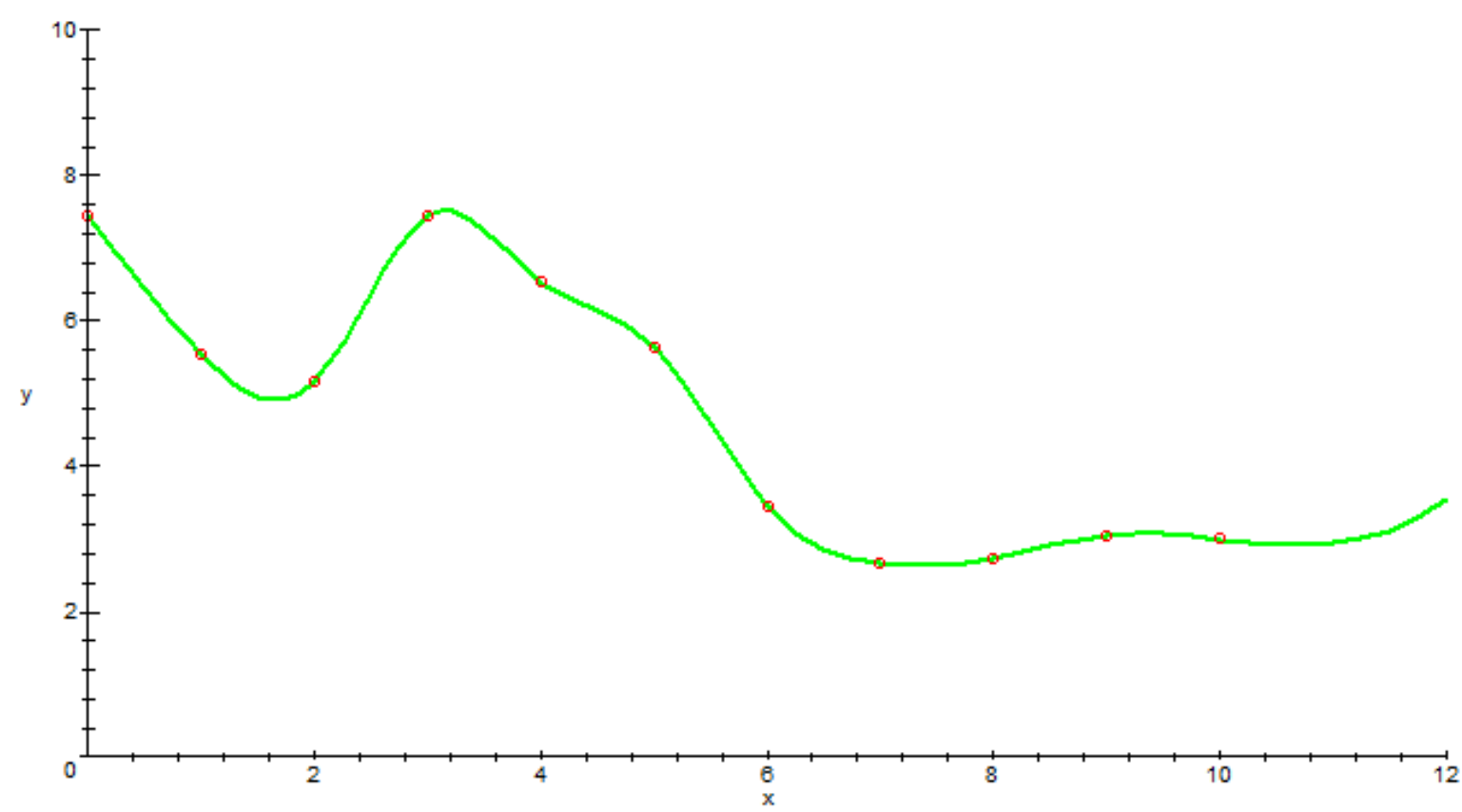

Figure 4 - The Spline function "Estimated".

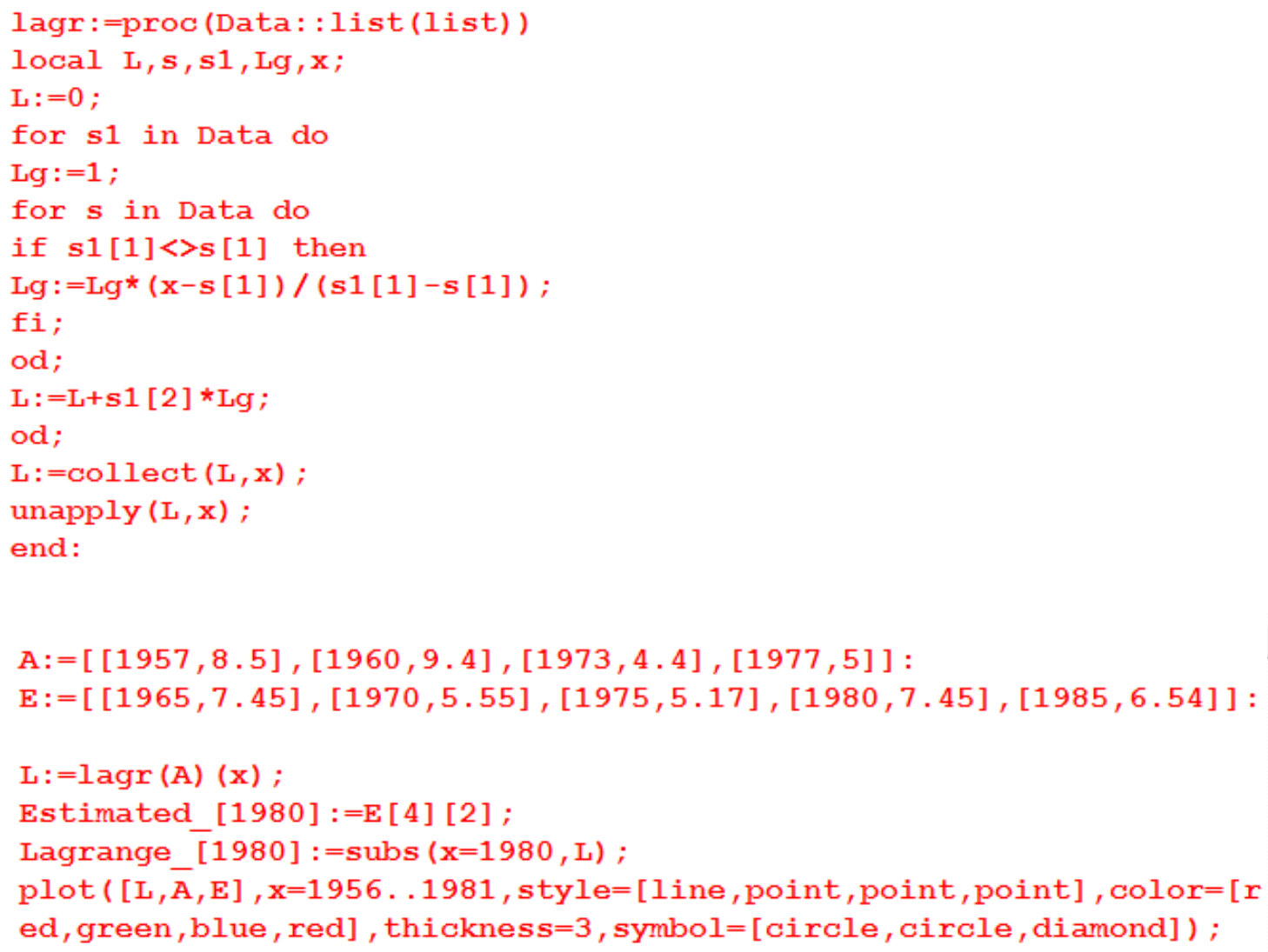




\begin{tabular}{l|lrl|l|ll} 
& ISRA (India) & $=\mathbf{1 . 3 4 4}$ & SIS (USA) & $=\mathbf{0 . 9 1 2}$ & ICV (Poland) & $=\mathbf{6 . 6 3 0}$ \\
Impact Factor: & ISI (Dubai, UAE) $=\mathbf{0 . 8 2 9}$ & PUHU (Russia) $=\mathbf{0 . 1 7 9}$ & PIF (India) & $=\mathbf{1 . 9 4 0}$ \\
& GIF (Australia) & $\mathbf{0 . 5 6 4}$ & ESJI (KZ) & $=\mathbf{1 . 0 4 2}$ & & \\
& JIF & $=\mathbf{1 . 5 0 0}$ & SJIF (Morocco) $=\mathbf{2 . 0 3 1}$ & &
\end{tabular}

$$
\begin{gathered}
L:=.150000000 x-291.550000 \\
\text { Estimated }_{-1980}=7.45 \\
\text { Lagrange }_{-1980}=5.4500000
\end{gathered}
$$

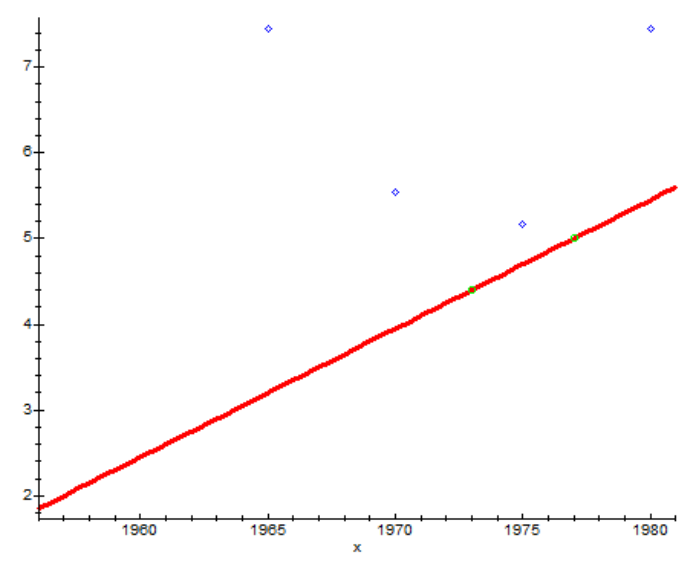

$$
\begin{gathered}
L=.003711821264 x^{3}-21.90541571 x^{2}+43091.25871 x-.282553046110^{8} \\
\text { Estimated_1980 }=7.45 \\
\text { Lagrange }_{-1980}=7.58
\end{gathered}
$$

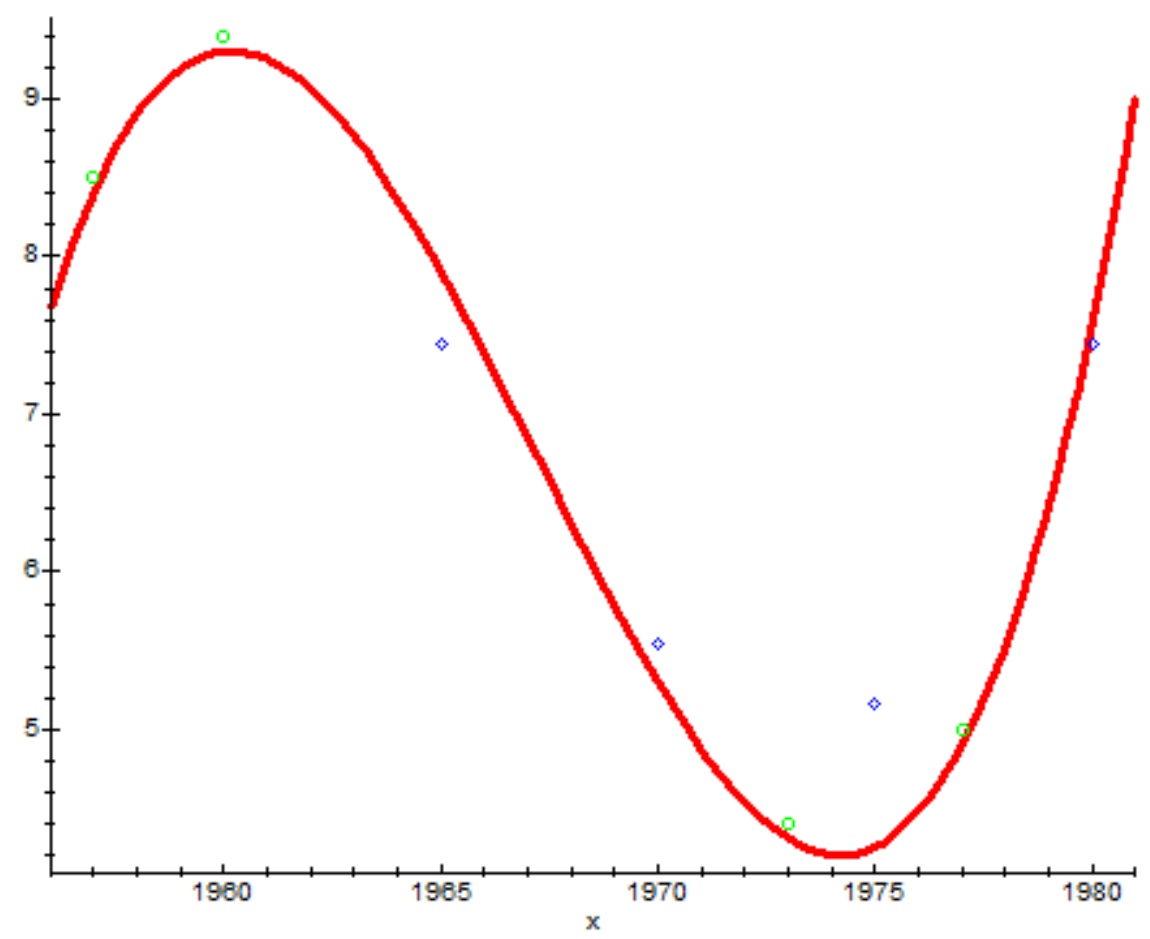

Figure 5 - Correct predictions "Estimated" on the first 4 points. 


\begin{tabular}{l|lrl|l|ll} 
& ISRA (India) & $=\mathbf{1 . 3 4 4}$ & SIS (USA) & $=\mathbf{0 . 9 1 2}$ & ICV (Poland) & $=\mathbf{6 . 6 3 0}$ \\
Impact Factor: & ISI (Dubai, UAE) $=\mathbf{0 . 8 2 9}$ & PUHU (Russia) $=\mathbf{0 . 1 7 9}$ & PIF (India) & $=\mathbf{1 . 9 4 0}$ \\
& GIF (Australia) & $\mathbf{0 . 5 6 4}$ & ESJI (KZ) & $=\mathbf{1 . 0 4 2}$ & & \\
& JIF & $=\mathbf{1 . 5 0 0}$ & SJIF (Morocco) $=\mathbf{2 . 0 3 1}$ & &
\end{tabular}

$L=-2.500000000 x+4963.5$

Estimated $_{-1985}=6.54$

Lagrange $_{-1985}:=1.000000$

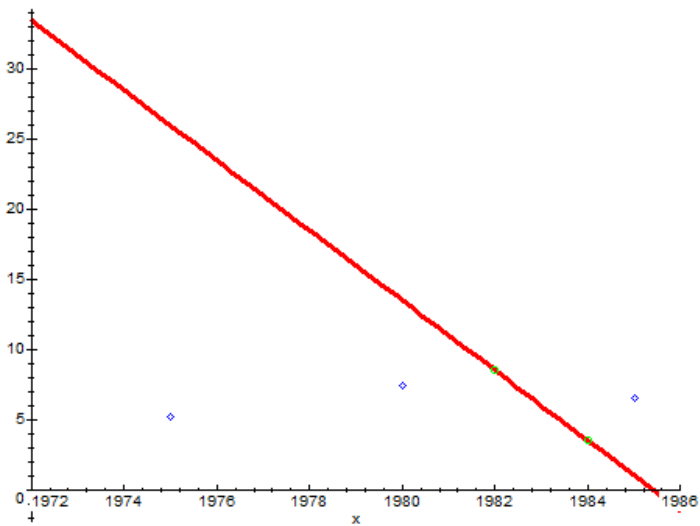

$L=-.04711399711 x^{3}+279.5413419 x^{2}-552865.8578 x+.364477843510^{9}$

Estimated_1985 $=6.54$

Lagrange $-1985=-2.8$

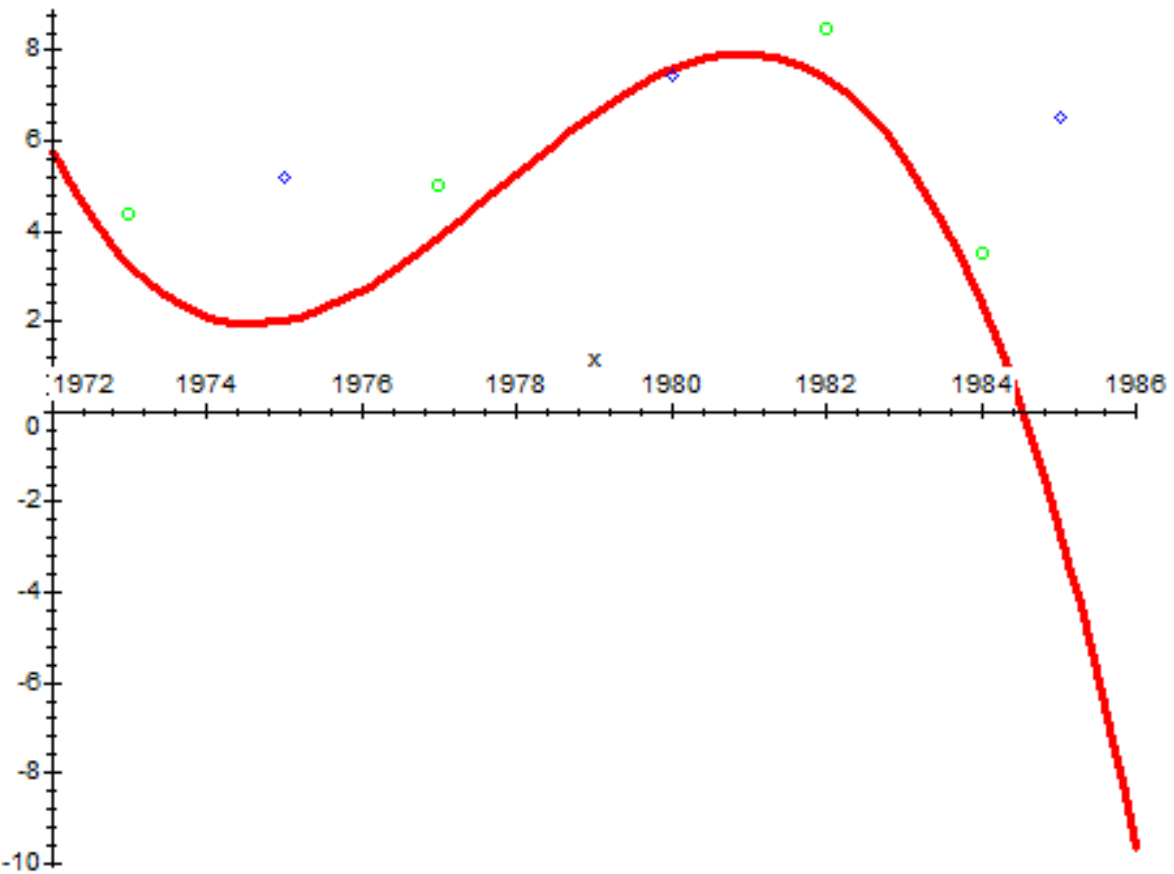

Figure 6 - Incorrect prediction "Estimated".

ISPC Technological advances, 


\begin{tabular}{l|lrl|l|ll} 
& ISRA (India) & $=\mathbf{1 . 3 4 4}$ & SIS (USA) & $=\mathbf{0 . 9 1 2}$ & ICV (Poland) & $=\mathbf{6 . 6 3 0}$ \\
Impact Factor: & ISI (Dubai, UAE) $=\mathbf{0 . 8 2 9}$ & PUHU (Russia) $=\mathbf{0 . 1 7 9}$ & PIF (India) & $=\mathbf{1 . 9 4 0}$ \\
& GIF (Australia) & $\mathbf{0 . 5 6 4}$ & ESJI (KZ) & $=\mathbf{1 . 0 4 2}$ & & \\
& JIF & $=\mathbf{1 . 5 0 0}$ & SJIF (Morocco) $=\mathbf{2 . 0 3 1}$ & &
\end{tabular}

$$
\begin{gathered}
L=-.1357142858 x^{3}+808.5871442 x^{2}-.160585559610^{7} x+.10630765541010 \\
\text { Estimated-1990 }=5.65 \\
\text { Lagrange }-1990=3 .
\end{gathered}
$$

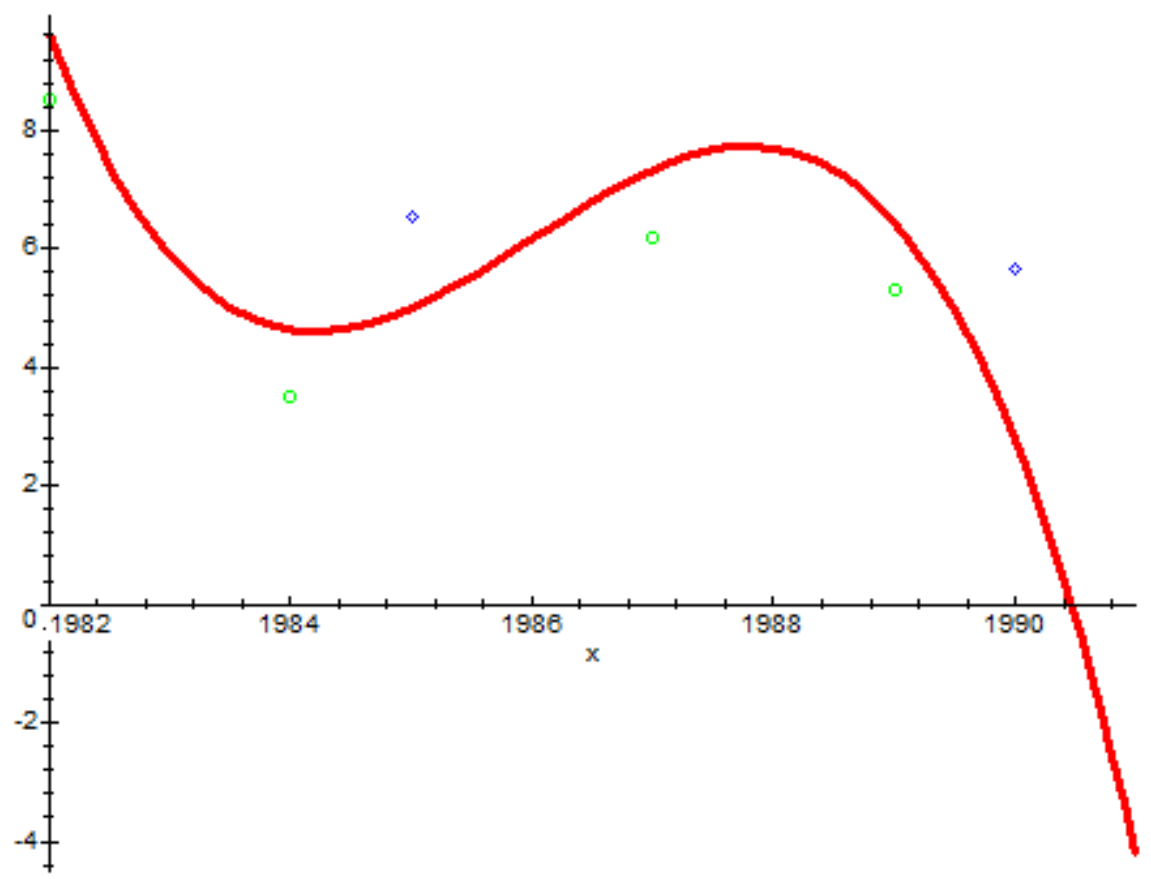

Figure 7 - Incorrect prediction "Estimated".

In conclusion we can say that the predictions were incorrect. Although the fourth point forecast and the same (Fig.5), but all the other points of interpolation of Lagrange 4 of the order are not applicable to predict (Fig.6-7) .

\section{References:}

1. Behzod Gaybullaev, Su-Chin Chen, Dilmurod Gaybullaev (2012) Changes in water volume of the Aral Sea after 1960. Appl Water Sci (2012) 2: 285-291. DOI 10.1007/s13201-012-0048-Z

2. Shevtsov AN, Egemberdieva SK (2012) Algoritmy interpolyatsii. Materialy respublikanskoy nauchno-prakticheskoy konferentsii magistrantov, doktorantov i molodykh prepodavateley na temu «Nauka i sovremennost' - 2012». II t. - Taraz: Taraz universiteti, 2012. pp.32-35.
3. Shevtsov AN, Dzhunisbekov TM, Dzhakiyaev DK, Yusupova LK (2012) Analiz grafikov relaksatsii napryazheniy $\mathrm{s}$ ispol'zovaniem Delphi 2011. Materialy MN-PK «Urkimbaevskie chteniya» «Vodnye resursy i puti ikh ratsional'nogo ispol'zovaniya $\mathrm{V}$ sovremennykh usloviyakh». - Taraz: Taraz universiteti, 2-3 nov. 2012. pp.306-310.

4. Shevtsov AN (2013) DOUBLE INDEXATION OF BINARY OPERATIONS ON THE GRAPHS Theoretical \& Applied Science№10, 2013. - pp.18-23. 


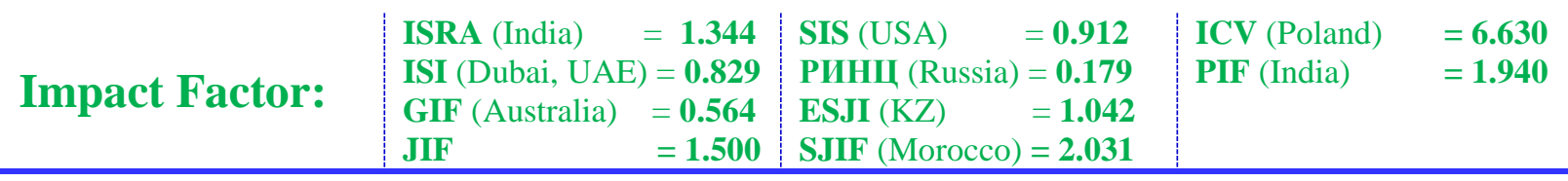

5. (2015) Interpolyatsiya polinomami Lagranzha i N'yutona. Available: http://www.machinelearning.ru/wiki/index.php? title=Interpolyatsiya_polinomami_Lagranzha_i N'yutona (Accessed: 10.11.2015).

6. (2015) Interpolyatsiya metodom Lagranzha. Available:

http://www.maple9.ru/ma07/Index1.htm (Accessed: 10.11.2015).

7. (2015) Interpolyatsiya metodom Lagranzha. Available: http://samoucka.ru/document23167.html (Accessed: 10.11.2015).

8. (2015) Interpolyatsionnyy mnogochlen Lagranzha - Maple. Available: http://www.cyberforum.ru/maple/thread138945 5.html (Accessed: 10.11.2015).

9. (2015) Interpolyatsiya polinoma Lagranzha Maple.

Available: http://www.cyberforum.ru/maple/thread695729. html (Accessed: 10.11.2015). 


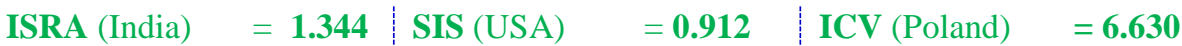

Impact Factor:

ISI (Dubai, UAE) $=\mathbf{0 . 8 2 9}$

GIF (Australia) $=\mathbf{0 . 5 6 4}$

РИНЦ (Russia) $=0.179$

PIF $($ India) $\quad=1.940$

JIF

$=1.500$

$\mathrm{ESJI}(\mathrm{KZ}) \quad=1.042$

SJIF $($ Morocco $)=\mathbf{2 . 0 3 1}$ 